\title{
Automatic Lung Tumor Detection Based on GLCM features
}

\author{
Mir Rayat Imtiaz Hossain, Imran Ahmed, Md. Hasanul Kabir \\ Department of Computer Science and Engineering, \\ Islamic University of Technology, Gazipur, Bangladesh
}

\begin{abstract}
For diagnosis of lung tumors, CT scan of lungs is one of the most common imaging modalities. Manually identifying tumors from hundreds of CT image slices for any patient may prove to be a tedious and time consuming task for the radiologists. Therefore, to assist the physicians we propose an automatic lung tumor detection method based on textural features. The lung parenchyma region is segmented as a preprocessing because the tumors reside within the region. This reduces the search space over which we look for the tumors, thereby increasing computational speed. This also reduces the chance of false identification of tumors. For tumor classification, we used GLCM based textural features. A sliding window is used to search over the lung parenchyma region and extract the features. Chi-Square distance measure is used to classify the tumor. The performance of GLCM features for tumor classification is evaluated with the histogram features.
\end{abstract}

\section{Introduction}

Lung cancer is the leading cause of cancer death all over the world [3]. The prevalence of lung cancer is only second to breast cancer in women and prostate cancer in men. About $80 \%$ of the lung cancer patients' present advanced-stage disease (stages III and IV) and are considered inoperable due to loco-regional tumor extension, extra thoracic spread or poor physical condition at the time of diagnosis [4]. If detected at an early stage, life of a cancer patient can be saved.

Manually detecting tumors from CT images of the lungs can be a tedious and time consuming task as the radiologists may have to go thorugh hundreds of slices to predict the tentative tumorous regions. Automating the process of tumor detection would assist the physicians to detect tumors tentatively in a much quicker time without having to go through each of the hundred slices manually. However, automating the process is challenging, due to high diversity in appearance of tumor tissue among different patients and in many cases, similarity between tumors and normal tissues becase of the low contrast in CT images [1, $2]$.

Computed Tomography is one of the best imaging techniques for soft tissue imaging behind bone structure [5]. A modern multislice CT machine enables the rapid acquisition of precise sets of successive images with very high resolution, 
supporting a more valid diagnosis. CT images help to detect and locate pathological changes. Thus, our purpose is to automate the detection of tumorous regions from $\mathrm{CT}$ images.



Fig. 1. CT Image of Lungs.

Because of the ability of the PET-CT scans to determine the stage of tumors and the high contrast images they produce, many literature works are based on PET CT images. In their paper, Kanakatte et. al. [6] proposed an automatic lung tumor segmentation from PET CT images using standardized uptake values (SUV) and connected component analysis. Cherry Ballangan et. al. [7] proposed a tumor-customized downhill method which automatically formulated a tumorcustomized criterion function for improving tumor boundary definition and then used a monotonic property of the standardized uptake value (SUV) of tumors to separate the tumor from adjacent regions of increased metabolism. Hui Chui et. al. [8] used neighbourhood grey-tone difference matrix (NGTDM) to calculate contrast features of PET volume in SUV to automatically localize tumor and then based on analyzing the surrounding CT features of the initial tumor definition a decision rule was devised. Although, the ability of conventional CT scan to accurately determine tumors and its stages is limited in comparison to imaging techniques such PET CT imaging which uses radioactive materials like Fluro deoxy-glucose (FDG), CT scan is much cheaper and easily obtainable than PET CT images. 
There were several approaches that used semi-automatic click and grow techniques. Rios Velazquez et. al. [9] proposed a semi-automatic click and grow algorithm for detecting lung lesions from PET CT images. Plajer and Richer [10] proposed a new active contouring algorithm [12] for lung tumor segmentation in $\mathrm{CT}$ images where the user needs to specify the initial contours.

Amongst approaches that involved texture analysis Malone et. al. [11] proposed a method for identifying diseases in CT image of lungs using 18 textural features and the classification is done using Support Vector Machine (SVM). Wei and $\mathrm{Hu}$ [13] used GLCM [17] and GLRLM [19] features for identifying Lobar fissure from Lung CT images using Neural Network. A comparison of using GLCM, GLRLM and wavelet features for segmenting brain tumors from CT images was done by Padma and Sukanesh [14]. Same authors, in their another work[15], used a combination of wavelet transform with GLCM features for brain tumor segmentation. Kadi and Watson [16] proposed a method for differentiating between aggressive and non-aggressive lung tumor using fractal analysis, but the tumorous regions were manually segmented. Although GLCM, GLRLM has been used for brain tumor detection, lobar fissure detection, etc. only a few works has been done to detect lung tumor from CT images using texture analysis.

In this paper, for tumor detection, we are using texture analysis technique. Image texture gives us information about the spatial arrangement of color or intensities in an image or selected region of an image. For texture anlysis, we are using GLCM textural features. We choose GLCM features because it gives us texture pairs with matching second order statistics, which cannot be discriminated by human eye [1]. However, due to textural similarities between normal tissues and tumor, GLCM features alone cannot properly detect tumorous regions. It might yield into lots of false positive values. Therefore, we need to perform our search only within those regions where there is probability of tumor, namely within the lung parenchyma. For this, we segment the lung parenchyma first and then extract the GLCM features. One of the major challenges is to identify tumours that appear to be attached to the chest wall. So segmenting the lung parenchyma properly is an important factor in determining tumor correctly.

One of the major advantages of our approach compared to other approaches is that our method is automatic and does not require the user to specify any seed point or region of interest. Tumorous regions and nodules that are to be detected are found within the lung parenchyma, an area which encompasses only about half of the area of the computed tomography (CT) image slice. Since we are segmenting the lung parenchyma beforehand, the space over which we search for tumors is reduced. So the processing time would be reduced significantly since we would now search only within a specific area. Moreover, the number of false positives would be considerably lower if the lung parenchyma is segmented beforehand. 


\section{Dataset Description}

The CT images of the lungs were collected from Popular Diagnostic Center, Bangladesh. CT scans from 18 patients were collected out of which, 12 were male and 6 were female. All of these patients had tumors and were within an age group of 23 to 77 . The slice thickness for the CT scan was $5 \mathrm{~mm}$. The image slices were isotropic and had a resolution of $512 \times 512$. For a person with average lung size, an image stack contains more than 200 images.

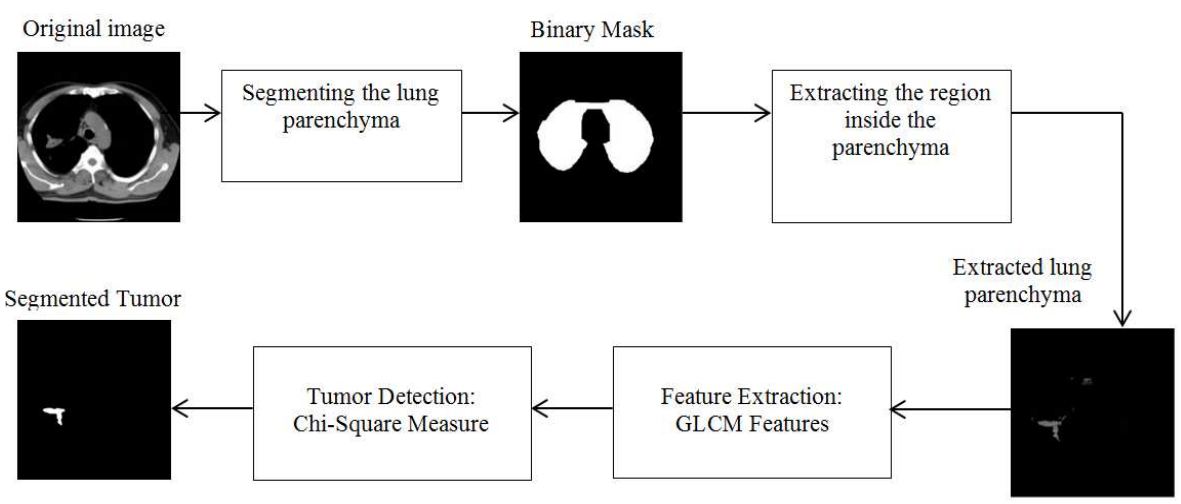

Fig. 2. Overview of our proposed method.

\section{Proposed Technique}

We proposed a GLCM feature based approach for the detection of lung tumors. First, we segment out the lung parenchyma, using morphological operations, because this is the region where all pathological changes take place. This reduces the search space and also reduces the chance of false detection of tumors because of the textural similarity with some portion of the chest wall and the heart. Once, lung parenchyma is segmented we extract the GLCM features and classify the tumor using a minimum distance classifier. Fig. 2 shows the overview of our process illustrating four phases:

- Segmentation of lung parenchyma

- Feature Generation

- Training Phase

- Classification phase

\subsection{Segmentation of the lung parenchyma}

Due to the textural similarities between the tumors, chest walls and the heart GLCM features alone cannot discern them properly. Therefore, proper segmentation of lung parenchyma is important as the tumors reside within these regions. 

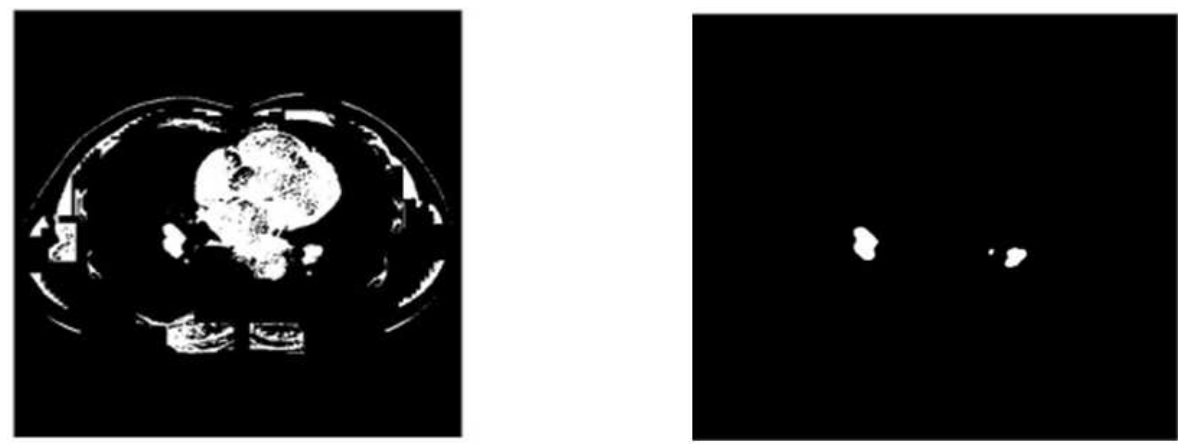

Fig. 3. Classification using GLCM only without segmenting the lung (Left), Proposed method using GLCM(Right).

This reduces the space over which we search for the tumor massively as lung parenchyma covers only about half the area of CT image. Moreover, it gets rid of chest walls and the heart and thereby reduces the chance of false positive dections. Fig. 3 shows the necessity of segmentation of lung parenchyma segmentation.

From the CT image of lungs it can be observed that the the regions corresponding the lung parenchyma are dark. Therefore, we invert the image and perform global adaptive thresholding [18]. To remove salt and pepper noise that are created due to the thresholding, a $5 \times 5$ median filter is used. Once the noise is removed, the connected components are extracted. The largest connected component is removed since it represents the background. The next two largest connected components are kept as they represent the two sides of lung parenchyma. Sometimes due to low contrast in images, the two sides of the lung seem to be attached. In that case, we keep the largest remaining connected component. Since tumors are present within the lung parenchyma they cause holes or intrusions within the large white regions. To fill the holes or intrusions we perform dilation $k$ times and erosion $k+1$ times where the value of $k$ was emperically determined to be $k=10$. We ran erosion one time more than dilation to ensure that no part of chest wall or heart falls within the segmented lung parenchyma. Fig. 4 shows the result of lung parenchyma segmentation.

In some of the images, the chest wall touches the border of the image. In such cases, the exterior region of the lungs would no longer remain a connected to component. To connect the background, one pixel from each border is padded with zero value. Fig. 5 shows the effect of padding zero valued pixels.

\subsection{Feature Generation}

\section{GLCM Features:}

Gray Level Co-ocurence Matrix (GLCM) [17] is used to extract the second order statistical texture features. The matrix denoted by $h_{d, \theta}(i, j)$ gives the 

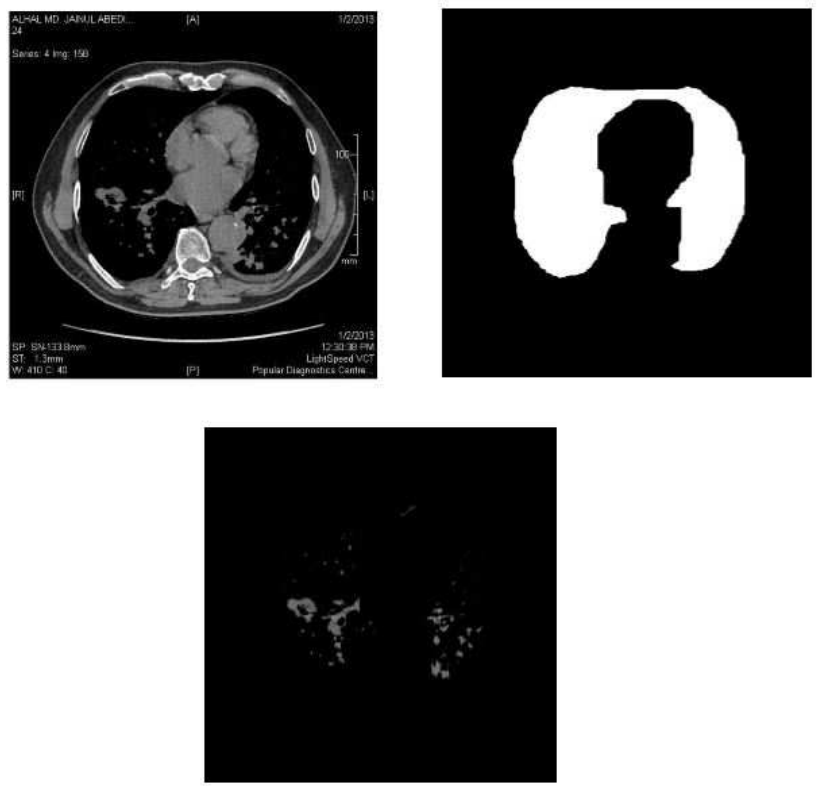

Fig. 4. Original Image (Top Left) , Binary mask corresponding the lung parenchyma (Top Right), After performing AND Operation (Bottom).
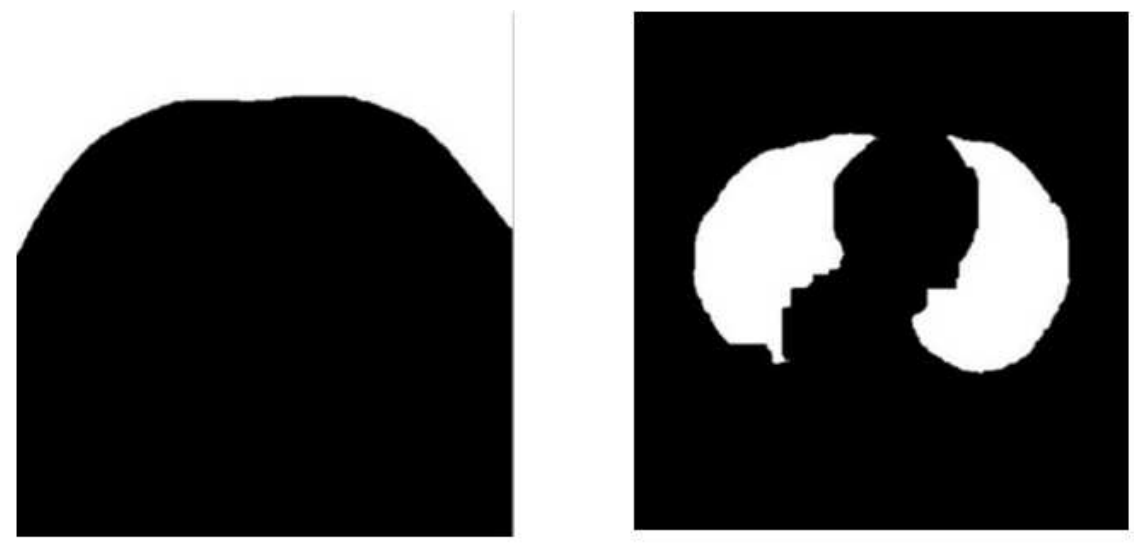

Fig. 5. Binary mask obtained without padding zero (Left), binary Mask obtained after padding zero (Right). 
number of times two pixels with gray level $\mathrm{i}$ and $\mathrm{j}$ co-occur at a distance $\mathrm{d}$ and an angle $\theta$. When divided by the total number of pixels in the image, this estimate $p_{d, \theta}(i, j)$ gives the joint probability of co-ocurence of a pair of pixels.

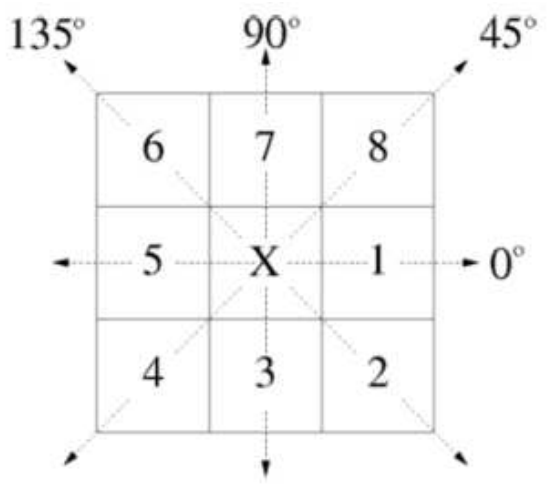

Fig. 6. Eight nearest neighbor pixels used to describe pixel connectivity. Cells 1 and 5 show the horizontal, 4 and 8 the right diagonal, 3 and 7 the vertical and 2 and 6 the left diagonal nearest neighbors.

Four directions are required to describe the texture content in the horizontal $0^{0}$, vertical $90^{\circ}$, right $45^{\circ}$ and left-diagonal $135^{0}$ [1] as shown in Fig. 6. A complete representation of image texture is contained in the co-occurrence matrices calculated in these four directions. Extracting information from these matrices using textural features, which are sensitive to specific elements of texture, provides unique information on the structure of the texture being investigated. Fig. 7 shows an example of the calculation of a horizontal co-occurence matrix (at $0^{0}$ ) on a $4 \times 4$ image containing four gray levels from 0 to 4 .

\begin{tabular}{|l|l|l|l|}
\hline 0 & 2 & 2 & 2 \\
\hline 0 & 3 & 2 & 2 \\
\hline 1 & 2 & 2 & 2 \\
\hline 3 & 0 & 3 & 0 \\
\hline
\end{tabular}

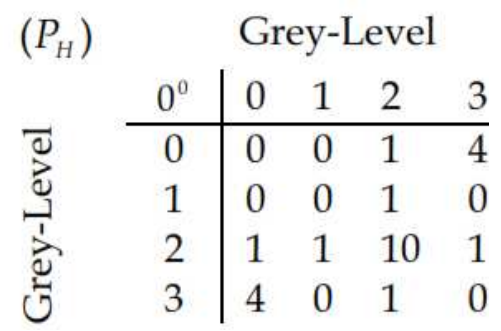

Fig. 7. Simple example demonstrating the formation of a co-occurrence matrix from an image. Left $4 \times 4$ image with four unique grey-levels.Right, the resulting horizontal co-occurrence matrix. 
Given an image $f(x, y)$, indicating the pixel intensity value at position $(x, y)$, with a set of $\mathrm{G}$ discrete intensity levels, matrix $h_{d, \theta}(i, j)$ is defined in such a way that its $(i, j)$ th entry is equal to the number of times $f\left(x_{1}, y_{1}\right)=i$ and $f\left(x_{2}, y_{2}\right)=j$ where $\left(x_{1}, y_{1}\right)$ and $\left(x_{2}, y_{2}\right)$ are two pixels such that $\left(x_{2}, y_{2}\right)=$ $\left(x_{1}, y_{1}\right)+(d \cos \theta, d \sin \theta)$. This yields a square matrix of dimension equal to the number of intensity levels in the image, for each distance $\mathrm{d}$ and orientation $\theta$. The classification of fine textures requires small values of $d$, whereas coarse textures require large values of $d$. Reduction of the number of intensity levels (by quantizing the image to fewer levels of intensity) helps increase the speed of computation, with some loss of textural information.
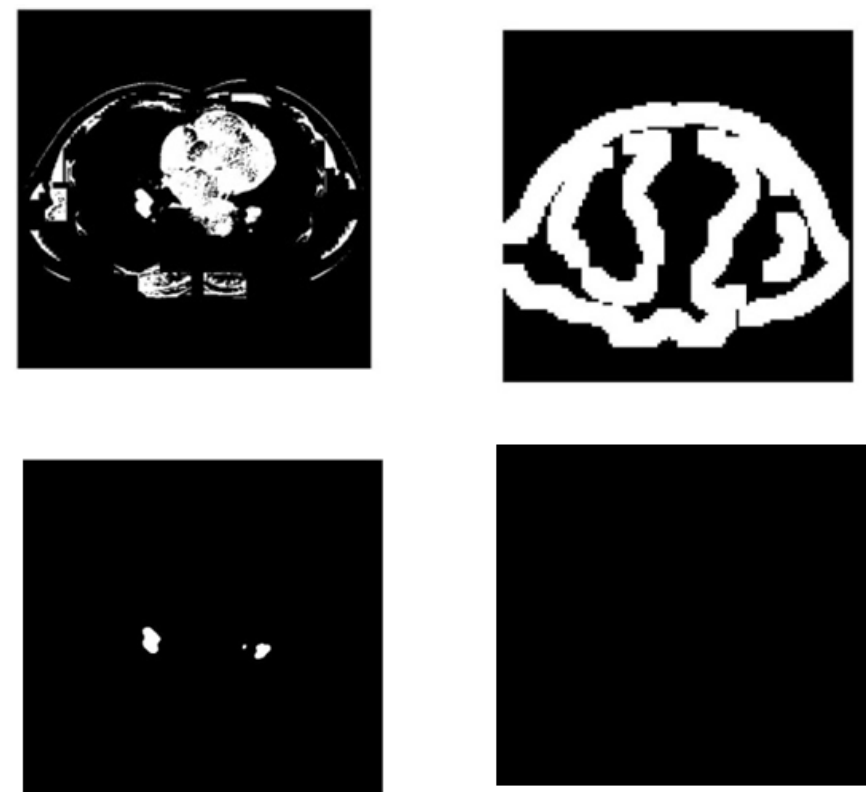

Fig. 8. Using 10 GLCM features (Top Left) without segmenting lung parnechyma, using all the 14 GLCM features (Top Right) without segmenting lung parenchyma, proposed method using 10 GLCM features (Bottom Left), using all 14 GLCM features (Bottom Right).

Haralick et al. proposed a set of 14 local features specifically designed for this purpose [17]. In the table we list the features in Table 1. However out of these 14 features we selected 10 features by the method of backward search algorithm [1]. The four features which were not selected are Sum of Squares: Variance, Sum Average, Sum Variance and Maximal Correlation Coefficient. Fig. 8 shows the result of using all GLCM features for classification and how selection of 10 featuers make the result better. 
Table 1. List of GLCM features

\begin{tabular}{ll}
\hline Sl No. & Features \\
\hline 1. & Angular Second Moment \\
2. & Contrast \\
3. & Correlation \\
4. & Sum of Squares: Variance \\
5. & Inverse Difference Moment \\
6. & Sum Average \\
7. & Sum Variance \\
8. & Sum Entropy \\
9. & Entropy \\
10. & Difference Entropy \\
11. & Difference Variance \\
12. & Information measures of Correlation I \\
13. & Information measures of Correlation II \\
14. & Maximal Correlation Coefficient \\
\hline
\end{tabular}

\subsection{Training Phase}

For the purpose of training we took 50 patches of tumorous regions as the training set. From these images 10 GLCM features were extracted. In order to generate the GLCM Matrix, $\mathrm{Q}=16$ quantization levels were taken where the 256 grey levels are quantized to 16 grey levels. For each of the training samples, four GLCM matrices were generated for the four directions $0^{0}, 45^{\circ}, 90^{\circ}$ and $135^{\circ}$ and a pixel distance of 1 pixel. We calculated the features for each of the four directions and took the average of them. Then the mean feature vector for the training images was calculated. We also applied adaptive thresholding [18] on each of the tumor image to find the maximum and the minimum grey level values of the tumors.

\subsection{Classification phase}

For the purpose of testing 54 images were used, 3 slices from each of the 18 patients. All of these patients had tumors. Classification phase begins by first segmenting out the lung parenchyma of the test image. It is because tumors reside within the lung parenchyma and this reduces the space over which we search for tumor. To search through the segmented lung parenchyma, we have used a sliding window of dimension $30 \times 30$ which is slided by 5 pixels in each iteration. If all the pixels within the window are black, the window is simply slided without computing any features within it. Otherwise, the GLCM features within the region are calculated. Then, using chi-square distance measure, the distance from the feature vector of the window to the mean vector is measured. The equation of Chi-Square is given by:

$$
\chi^{2}=\sum_{j=1}^{J} \frac{(S(j)-M(j))^{2}}{S(j)+M(j)}
$$




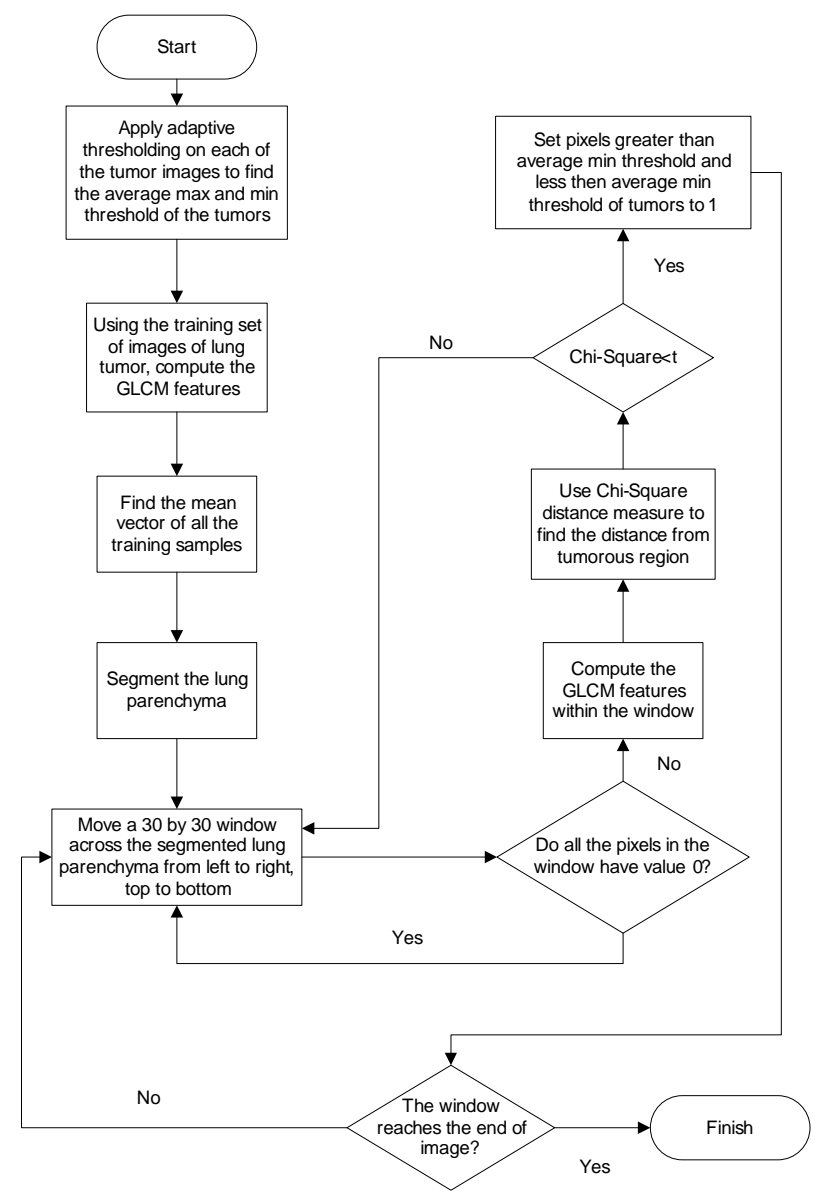

Fig. 9. Flowchart of the tumor identification process. 
Where, $\chi^{2}=$ Chi-square measure, $J=$ No. of features,$M(j)=j$ th feature of mean vector and $S(j)=j$ th feature of the test image patch.

If the chi-square measure is less than a certain threshold value, $t$, which is determined emperically, then we can deduce that the window belongs to the tumorous region. However, some part of the window might have non-tumorous pixels. So the pixels which have a gray level intensity within the min and the max threshold value of the tumors are considered to be tumorous.

Chi-Square methods is preferred over other classifiers like SVM because it is computationally faster and simple, yet produces desirable result for us. The description of our classification process is represented with a flowchart as shown in Fig. 9.

\section{Results}

We evaluated the performance of our method using GLCM features against the histogram features. For evaluating our results we used Precision, Recall and Fmeasure. In pattern recoginition and information retrieval, precision (also called positive predictive value) is the fraction of retrieved instances that are relevant, while recall (also known as sensitivity) is the fraction of relevant instances that are retrieved. Both precision and recall are therefore based on an understanding and measure of relevance. Precision can be seen as a measure of exactness or quality, whereas recall is a measure of completeness or quantity. In simple terms, high recall means that an algorithm returned most of the relevant results, while high precision means that an algorithm returned substantially more relevant results than irrelevant. The precision and recall is defined as:

$$
\begin{gathered}
\text { Precision }=\frac{T P}{T P+F P} \\
\text { Recall }=\frac{T P}{T P+F N}
\end{gathered}
$$

F-measure is the harmonic mean of precision and recall and is given by:

$$
\text { Fmeasure }=\frac{2 * \text { precision } * \text { recall }}{\text { precision }+ \text { recall }}
$$

We implemented our method in MATLAB and executed on Intel Core 2 Duo 2.67 $\mathrm{GHz}$ processor with 4.00 GB RAM.

In Fig. 10 we show some of the results that we obtained and compared them with the ground truth. The ground truth were labelled by a radiologist. We can see from Fig. 10 that our method was able to detect tumors properly in most cases, even the ones adhering to the chest walls, heart and pleura.

\subsection{Detection rate of GLCM features}

After evaluating our method using GLCM features with histogram features we found out that GLCM features gives us a precision of $85.5 \%$, recall of $91 \%$ and 

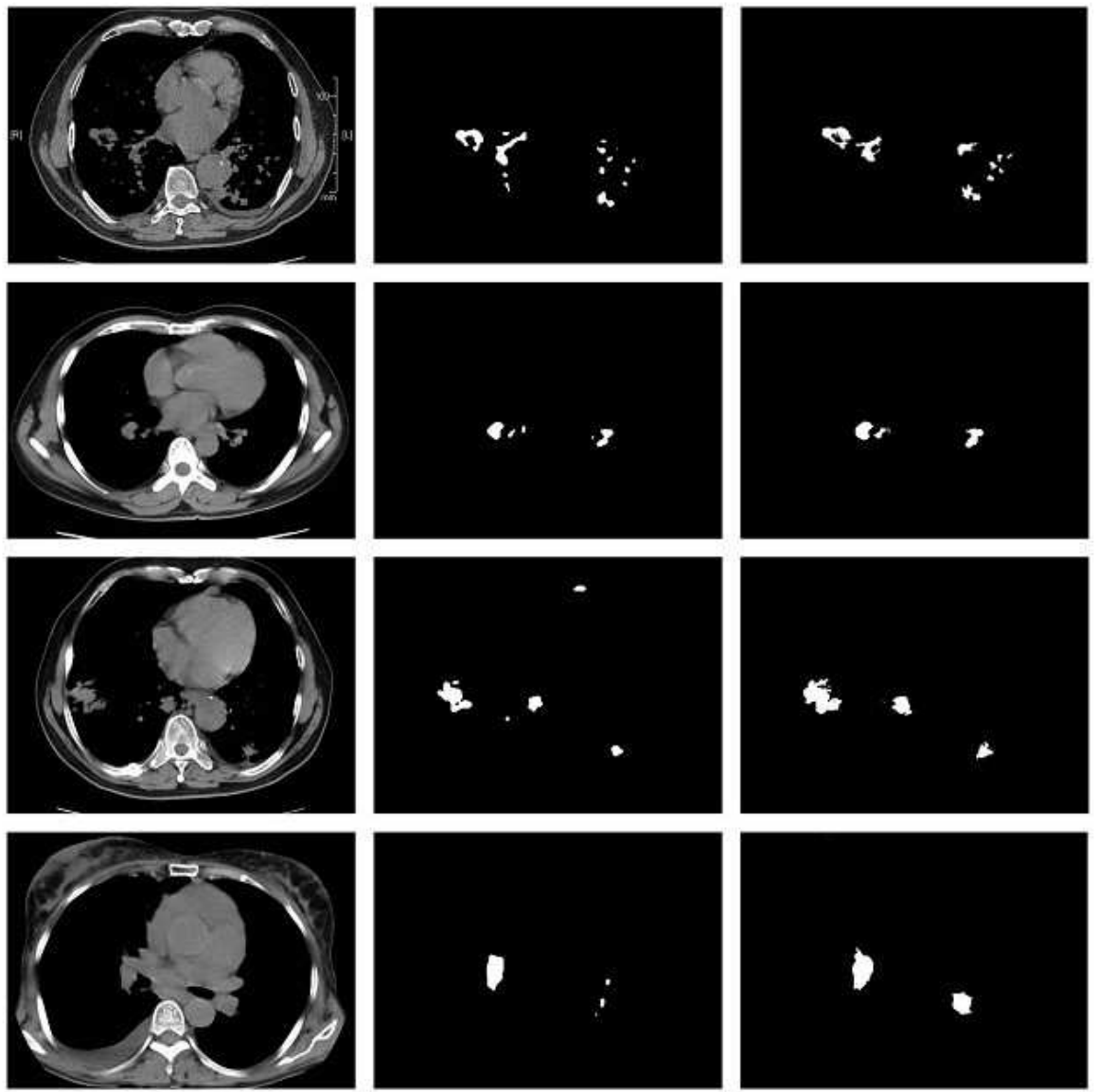

Fig. 10. Original Image (Left), Result of proposed method (center), Ground truth (right). 
f-measure of $88 \%$ compared to a precision of $83.7 \%$, recall of $85.1 \%$ and a $\mathrm{f}$ measure of $84.4 \%$ for histogram features. The result is shown in Fig. 11.

Although we found that GLCM gives comparativelty better result than the histogram features, it is computationally slower than the histogram feautes. Histogram features took 5.89 seconds on average. On the other hand GLCM features took 26.57 seconds on average.

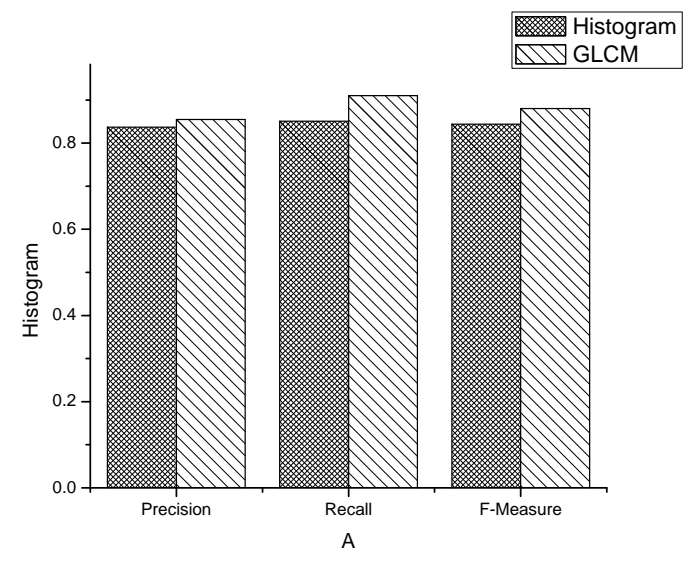

Fig. 11. Result comparison between Histogram and GLCM features.

\section{Conclusion}

Unlike, previous methods which require the user to specify either the seed points of tumorous regions or the some region of interest, our method can detect tentative tumorous regions automatically. Moreover, since we are segmenting the lung parenchyma initially, our search space is reduced thereby reducing the computational time. Also, morphological operations and GLCM features are computationally fast to compute. Because of the similarity in the texture between tumors and chest walls it is tough to get a desirable result by applying only texture analysis method. Therefore, accurate segmentation of lung parenchyma is an essential task.

\section{References}

1. Nailon, W.H.: Texture Analysis Methods for Medical Image Characterisation. Biomedical Imaging InTech (2010) 75-100

2. Castellano, G., Bonilha, L., Li, L.M., Cendes, F.: Texture analysis of medical images. Clinical Radiology 59 (2004) 1061-1069 
3. Jemal, A., Siegel, R., Xu, J., Ward, E.: Cancer statistics. CA Cancer J Clin. 60 (2010) 277-300

4. Auperin, A., Le Pechoux, C., Rolland, E., et al.: Meta-analysis of concomitant versus sequential radiochemotherapy in locally advanced non-small-cell lung cancer. J Clin Oncol. 28 (2010) 2181-2190

5. von Schulthess, G.K., Steinert, H.C., Hany, T.F.: Integrated PET/CT-3: Current applications and future directions. Radiology 238 (2006) 405-422

6. Kanakatte, A., Gubbi, J.,Mani, N., Kron, T., Binns, D.: A Pilot Study of Automatic Lung Tumor Segmentation from Positron Emission Tomography Images using Standard Uptake Values. IEEE Sysmposium on Computational Intelligence In Image and Signal Processing (CIISP) (2007) 363-368

7. Ballangan, C., Wang, X., Fulham, M., Eberl, S., Yin, Y., Feng, D.: Automated delineation of lung tumors in PET images based on monotonicity and a tumorcustomized criterion. IEEE Trans. on Information Technol Biomed. 15 (2011) 691702

8. Cui, H., Wang, X., Feng, D.: Automated localization and segmentation of lung tumor from PET-CT thorax volumes based on image feature analysis. Conf Proc IEEE Eng Med Biol Soc. (2012) 5384-5387

9. Rios Velazquez, E., Aerts, H.J., Gu, Y., Goldgof, DB., De Ruysscher, D., Dekker, A., Korn, R., Gillies, R.J., Lambin, P.: A semiautomatic CT-based ensemble segmentation of lung tumors: comparison with oncologists' delineations and with the surgical specimen. Radiotherapy and Oncology 105 (2012) 167-173

10. Plajer, I.C., Richter, D.: A new approach to model based active contours in lung tumor segmentation in 3D CT image data. Information Technology and Applications in Biomedicine (ITAB) (2012) 1-4

11. Malone, J., Rossiter, J.M., Prabhu, S., Goddard, P.: Identification of disease in CT of the lung using texture-based image analysis. Asilomar Conference on Signals, Systems and Computers 2 (2004) 1620-1624

12. Kass, M., Witkin, A., Terzopoulos, D.: Snakes: Active contour models. International Journal of Computer Vision 1 (1988) 321-331

13. Wei, Q., Hu, Y.: A study on using texture analysis methods for identifying lobar fissure regions in isotropic CT images. Conf Proc IEEE Eng Med Biol Soc. (2009) 3537-3540

14. Padma, A., Sukanesh, R.: Automatic Classification and Segmentation of Brain Tumor in CT Images using Optimal Dominant Gray level Run length Texture Features. International Journal of Advanced Computer Science and Applications(IJACSA) 2 (2011) 53-59

15. Nanthagopal, A.P., Sukanesh, R.: Wavelet statistical texture features-based segmentation and classification of brain computed tomography images. Image Processing, IET 7 (2013) 25-32

16. Al-Kadi, O.S., Watson, D.: Texture Analysis of Aggressive and Nonaggressive Lung Tumor CE CT Images. IEEE Transactions on Biomedical Engineering 55 (2008) 1822-1830

17. Haralick, R.M., Shanmugam, K., Dinstein, Its'Hak: Textural Features for Image Classification. IEEE Transactions on Systems, Man and Cybernetics SMC-3 (1973) 610-621

18. Gonzalez, R.C., Woods, R.E.: Digital Image Processing. Pearson Prentice Hall, Third Edition (2008)

19. Tang, X.: Texture information in run-length matrices. Image Processing, IEEE Transactions (1998) 7 1602-1609 